\title{
Parental perceptions of neighborhood processes, stress, personal control, and risk for physical child abuse and neglect ${ }^{\text {tr }}$
}

\author{
Neil B. Guterman ${ }^{\mathrm{a}, *}$, Shawna J. Lee ${ }^{\mathrm{b}}$, Catherine A. Taylor ${ }^{\mathrm{c}}$, Paul J. Rathouz ${ }^{\mathrm{d}}$ \\ a School of Social Service Administration and Chapin Hall Center for Children, University of Chicago, 969 East 60th Street, Chicago, IL 60637, USA \\ b School of Social Work and Merrill-Palmer Skillman Institute for Child and Family Development, Wayne State University, USA \\ c Tulane University School of Public Health and Tropical Medicine, Department of Community Health Sciences, USA \\ d Department of Health Studies, University of Chicago, USA
}

\section{A R T I C L E I N F O}

\section{Article history:}

Received 5 May 2009

Received in revised form 1 September 2009

Accepted 8 September 2009

Available online 8 November 2009

\section{Keywords:}

Neighborhoods

Parenting

Child abuse

Fragile Families and Child Wellbeing Study

Path analysis

\begin{abstract}
A B S T R A C T
Objective: This study set out to examine whether mothers' individual perceptions of their neighborhood social processes predict their risk for physical child abuse and neglect directly and/or indirectly via pathways involving parents' reported stress and sense of personal control in the parenting role.
\end{abstract}

Methods: In-home and phone interview data were examined cross-sectionally from a national birth cohort sample of 3,356 mothers across 20 US cities when the index child was 3 years of age. Mothers' perceptions of neighborhood social processes, parenting stress, and personal control were examined as predictors, and three subscales of the Parent-To-Child Conflict Tactics Scale (CTS-PC) were employed as proxies of physical child abuse and neglect risk. Structural equation modeling (SEM) was employed to test direct and indirect pathways (via parenting stress and control) from perceived neighborhood processes to proxy measures of physical child abuse and neglect. Multiple group SEM was conducted to test for differences across major ethnic groups: African American, Hispanic, and White.

Results: Although perceived negative neighborhood processes had only a mild direct role in predicting risk for physical child abuse, and no direct role on child neglect, these perceptions had a discernable indirect role in predicting risk via parenting stress and personal control pathways. Parenting stress exerted the clearest direct role on both physical abuse and neglect risk. This predictor model did not significantly differ across ethnic groups.

Conclusions: Although neighborhood conditions may not play a clear directly observable role on physical child abuse and neglect risk, the indirect role they play underscores the importance of parents' perceptions of their neighborhoods, and especially the role they play via parents' reported stress and personal control.

Practice implications: Such findings suggest that targeting parents' sense of control and stress in relation to their immediate social environment holds particular potential to reduce physical child abuse and neglect risk. Addressing parents' perceptions of their neighborhood challenges may serve to reduce parenting risk via improving parents' felt control and stress.

(c) 2009 Elsevier Ltd. All rights reserved.

\footnotetext{
is This research was supported by grant \#R01 HD41141-02 from the National Institute of Child Health and Human Development and the National Institute of Mental Health, and grant \#R49-CE000915-02 from the Centers for Disease Control and Prevention.

* Corresponding author.
} 
Neighborhoods have long been thought to play an important role in the etiology of physical child abuse and neglect, and often pose a central target of concern for policymakers and practitioners aiming to reduce the problem. An ecological framework points out that risk for physical child maltreatment can be understood as deriving from a nested array of influences in a family's social ecology, including those from macro- (e.g., socioeconomic and cultural), meso- (e.g., community and neighborhood influences), and micro-systems (within the parent-child dyad) (Belsky, 1984; Garbarino, 1977). Physical child abuse, and especially child neglect, are linked with economic impoverishment (National Research Council, 1993), and early work conducted by Garbarino and colleagues suggested that neighborhood social conditions may play a central role in determining the ways in which economic impoverishment influences risk for physical child abuse and neglect. In two related studies, Garbarino and colleagues compared neighborhoods matched socioeconomically and attributed observed differences in rates of reported child maltreatment to what they termed neighborhood "social impoverishment" (Garbarino \& Kostelny, 1992; Garbarino \& Sherman, 1980). Although groundbreaking in drawing attention to neighborhood social processes linked with risk for physical child maltreatment, these early post hoc matched comparison studies were not able to tease apart to what degree neighborhood factors may uniquely shape risk for physical child maltreatment, independent of broader economic factors (e.g., neighborhood-level poverty), and independent of individual family characteristics.

More recent work by Coulton, Crampton, Irwin, Spilsbury, and Korbin (2007) and Coulton, Korbin, Su, and Chow (1995) has drawn a distinction between neighborhood structural factors, such as local economic conditions, and neighborhood process factors, such as "community social disorganization" characterizing social patterns of interactions among residents and institutions within a neighborhood. Coulton and colleagues work overlaps conceptually with that of Robert Sampson and colleagues, who have posited the particular importance of neighborhood processes that purvey a sense of social cohesion and mutual trust among neighbors, as well as informal social control, or a sense of norms regarding appropriate behavior, jointly termed neighborhood "collective efficacy" (Sampson, Morenoff, \& Gannon-Rowley, 2002). Importantly, high levels of perceived neighborhood collective efficacy appear to serve an inhibitory role with respect to crime and intimate partner violence (Browning, 2002; Sampson, Raudenbush, Stephen, \& Earls, 1997), mediating the impact of economic disadvantage on the levels of interpersonal violence among residents of a neighborhood.

Specifically regarding risk for physical child abuse and neglect, Coulton, Korbin, and Su (1999) examined individuallevel factors and neighborhood-level structural and process influences in a multi-level modeling study, and reported a clear observable role for individual-level factors on child abuse potential, such as parents' perceived supports, their own education level, and their prior experiences with family violence. Although they also found a discernable but mild role for neighborhoodlevel impoverishment and child care burden on maltreatment risk (consistent with earlier work documenting the importance of neighborhood structural factors), they reported no significant remaining role for neighborhood-level process factors, such as residents' perceptions of community disorder or lack of control over children, after controlling for other individual- and family-level factors. Consistent with this set of findings, Molnar, Buka, Brennan, Holton, \& Earls (2003) reported that the unique predictive role of neighborhood-level process factors, such as the size of neighborhood social networks and aggregate measures of collective efficacy, did not play a discernable role in parent-to-child physical aggression, after accounting for family- and individual-level influences. These more carefully controlled studies have raised serious questions about the role that neighborhood-level social processes might directly play in child maltreatment risk, after accounting for important individual and family factors.

A somewhat separate line of inquiry has suggested that parents' individual perceptions of neighborhood processes may play a more discernable role in influencing parenting behaviors than objectively assessed neighborhood conditions (Furstenberg, Cook, Eccles, Elder, \& Sameroff, 1999; O’Neil, Parke, \& McDowell, 2001). Parents' perceptions of neighborhood processes have been shown to predict child behavior problems through indirect pathways on parents' psychological distress and depression, which in turn predict harsh parenting (Gutman, McLoyd, \& Tokoyawa, 2008; Kohen, Leventhal, Dahinten, \& McIntosh, 2005). Additionally, parents' perceptions of neighborhood processes have been shown to moderate or "buffer" the impact of harsh parenting on children's externalizing behavior (Silk, Sessa, Morris, Steinberg, \& Avenevoli, 2004). As yet, however, no empirical links have yet been reported identifying parents' individual perceptions of neighborhood processes as either direct or indirect predictors of risk for physical child abuse and neglect. The present empirical base, while suggesting that objective neighborhood-level processes may not exert a directly observable role on parents' physical abuse and neglect risk, does not clarify whether parents' individual perceptions of their neighborhoods may directly shape at-risk parenting, and/or whether such linkages may be indirectly mediated by factors such as parents' felt stress and sense of control in the parenting role. Parents' sense of control may serve as a likely mediator given that exposure to uncontrollable aversive neighborhood events (such as crime, vandalism, and other dangerous neighborhood conditions) has been shown to predict residents' reported stress and felt powerlessness (Geis \& Ross, 1998; Hill, Ross, \& Angel, 2005), along with numerous prior studies that have reported important links between parents' perceived stress and lack of personal control with risk for physical child abuse and neglect (Bugental \& Happaney, 2004; Bugental, Lewis, Lin, Lyon, \& Kopeikin, 1999; Haskett, Ahern, Ward, \& Allaire, 2006; Rodriguez \& Green, 1997; Rodriguez \& Richardson, 2007).

Given this, we set out to examine: (1) whether parents' individual perceptions of their neighborhood processes directly predict their physical child abuse and neglect risk and, (2) whether parents' individual perceptions of their neighborhood processes indirectly predict risk via pathways through parents' reported stress and sense of personal control in the parenting role. We examine these questions employing structural equation modeling to test these potential direct and indirect pathways in a national sample of families across 20 US cities to add to current knowledge regarding neighborhoods, parenting stress, and risk for physical child abuse and neglect. 
Table 1

Characteristics of mothers in the study sample.

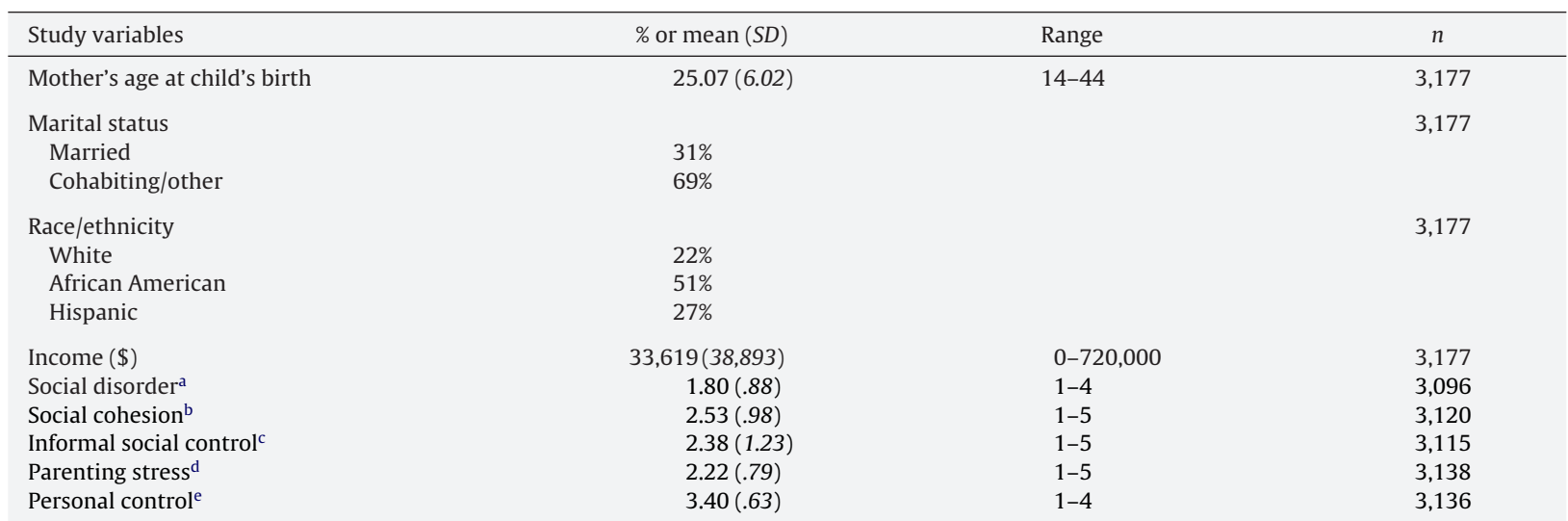

a Higher scores indicate higher levels of perceived social disorder.

b Higher scores indicate lower levels of perceived social cohesion.

c Higher scores indicate lower levels of perceived informal social control.

d Higher scores indicate greater parenting stress.

e Higher scores indicate greater personal control.

\section{Methods}

\section{Study design and procedures}

Analyses in the present study are drawn from the Fragile Families and Child Wellbeing Study (FFCWS), a longitudinal birth cohort study of families in 20 US cities with populations of 200,000 or more people, chosen by a stratified random sampling method to maximize cross-city variation of economic and policy environments (Reichman, Teitler, Garfinkel, \& McLanahan, 2001). The original FFCWS was designed to address broader questions about the conditions and capabilities of new parents and how their children fare over time, with focus placed on unwed parents. All study procedures were approved by Human Subjects Review Committees at Princeton and Columbia Universities. The original FFCWS birth cohort consisted of 4,898 index births in 75 hospitals with an oversampling of unwed families, including 3,712 births to unmarried mothers with 1,186 births to married mothers. Data were collected from parents at multiple time points, beginning at birth.

In the current study, we present cross-sectional analyses of mother-reported data obtained at the 3-year data collection point. At this point, a subset of FFCWS mothers who completed a phone interview when the index children were 3 years of age were invited to participate in a contiguous follow-up in-home interview. The in-home interview was designed to assess living conditions and the quality of the mother-child relationship that may indicate risk for physical child abuse and neglect. Data on child maltreatment proxies, parents' perceptions of their neighborhoods, parental stress, and parental sense of control were also collected during this assessment. Of the approximately 4,140 respondents who were eligible to participate in the in-home survey, 3,289 provided complete data on all study variables. Mothers who indicated their ethnicity as "other" $(n=112)$ were dropped because their small number did not permit subsample analyses across ethnic groups, resulting in a final sample of 3,177 mothers. Table 1 summarizes the descriptive statistics for the sample of mothers included in this study.

\section{Measures}

Perceived neighborhood processes were measured using three scales. The social disorder scale, a shortened version of that developed by Coulton et al. (1999) ( $\alpha=.93)$, employed eight questions that asked respondents to indicate how often parents assessed the following occurrences taking place in their neighborhood ranging from 1 (never) to 4 (frequently), including: Drug dealers or users hanging around; Drunks hanging around; Gang activities; Disorderly or misbehaving groups of teenagers. The construct was scored so that higher scores indicate a greater sense of perceived social disorder. The two scales comprising collective efficacy (Sampson et al., 1997) were used: (1) Informal social control was measured using five questions $(\alpha=.87)$ that asked respondents how likely they thought residents in their neighborhood would intervene in a series of situations (from $1=$ very likely to $5=$ very unlikely) such as: "Children were skipping school and hanging out on a street corner?" and "Children were showing disrespect to an adult?" These items were scored so that higher scores indicated lower levels of informal social control. (2) Social cohesion was measured with five questions $(\alpha=.79)$ that asked respondents to indicate their agreement (from $1=$ strongly agree to $5=$ strongly disagree) with statements such as: "People around here are willing to help their neighbors" and "People in this neighborhood can be trusted." Items were scored so that higher scores indicate a lower sense of social cohesion. A factor analysis indicated that these three neighborhood process scales (social disorder, informal social control, and social cohesion) did not represent separate factors, and were therefore combined into a single scale of perceived neighborhood processes $(\alpha=.90)$, with higher scores indicating more negative perceptions of neighborhood processes. 
Parenting stress was measured using a shortened version of the Parenting Stress Index (PSI) (Abidin, 1995) $(\alpha=.78)$. Mothers indicated how much they agreed (from $1=$ strongly disagree to $5=$ strongly agree), with six statements such as: "You find yourself giving up more of your life to meet your child(ren)'s needs than you ever expected" and "Since having (child) you feel that you are almost never able to do things that you like to do." Items were scored so that higher scores indicate greater parenting stress.

Personal control (Pearlin \& Schooler, 1978) was measured using five questions assessing mastery $(\alpha=.81)$. Mothers were asked to indicate their agreement (from 1 =strongly agree to $4=$ strongly disagree) with statements such as: "I have little control over the things that happen to me" and "There is little I can do to change many of the important things in my life." Items were scored so that higher scores indicate a greater sense of personal control.

To create latent constructs for structural equation models, scaled predictor variables were entered as the mean of item scores across all items on each scale (i.e., dividing the summed score of individual items by the number of items on each scale). Characteristics of all study predictor and control variables for the study sample are shown in Table 1.

Physical child abuse and neglect risk. To assess risk for future physical child abuse and neglect, we employed the Parent-Child Conflict Tactics Scales (CTS-PC) (Straus, Hamby, Finkelhor, Moore, \& Runyan, 1998). The CTS-PC measures mothers' selfreported acts of physical and psychological aggression directed toward the child, as well as instances of neglectful behavior, that occurred within the past year. The CTS-PC measure retains the basic conceptualization and operationalization of the original CTS scale, which has been used across multiple ethnic groups and in various nations with reported satisfactory psychometric properties including predictive validity, temporal consistency, and discriminant validity. We opted to use the CTS-PC as a primary indicator of physical child abuse and neglect risk over data from official protective services reports, given that protective services reports are based on investigatory practices holding substantial variability and discretion across workers, agencies, and state contexts (e.g., King, Reece, Bendel, \& Patel, 1998), and that individual worker decisions regarding filing a report are often subject to significant bias and inaccuracy (e.g., King et al., 1998; Socolar, Runyan, \& Amaya-Jackson, 1995).

The psychological aggression subscale of the CTS-PC $(\alpha=.48)$ was measured using five items assessing how many times mothers engaged in verbal and symbolic acts likely to cause psychological pain or fear in their child, including yelled or screamed at child, swore or cursed at him/her, and threatened to spank or hit but did not actually do it. The physical aggression subscale $(\alpha=.53)$ asked about a range of physically aggressive mother-to-child behaviors including how many times she shook the child, pinched him/her, slapped him/her on the head, face or ears, and hit him/her on the bottom with a hard object. Items measuring the most severe acts of physical aggression on this scale with rare base rates (e.g., "threatened with a knife or gun" or "burned or scalded on purpose") were dropped, as required by one Institutional Review Board. The neglect subscale $(\alpha=.50)$ employed five items that assessed how often, for example, the mother left the child home alone but thought some adult should be with him/her, was so caught up with her own problems that she was unable to show love to the child, and was not able to make sure the child got the food he/she needed. The modest alpha coefficients obtained in the present study are consistent with prior studies employing the CTS-PC scales, and are expected given their measurement of low base rate events exhibiting skewed distributions (Straus et al., 1998). All three scales were originally coded ordinally, with $0=n o n e$, 1 once, $2=$ twice, $3=3-5$ times, $4=6-10$ times, $5=11-20$ times, $6=$ more than 20 times. For the purposes of creating latent constructs for structural equation models, the individual items were recoded dichotomously $(0=$ none, $1=$ one or more times in the past year). We conducted comparative analyses of the measurement and structural models using both the original ordinal and dichotomous coding of CTS-PC variables, and results indicated that both the model fit and the individual path coefficients were essentially equivalent regardless of the coding scheme of the CTS-PC items. Therefore, we opted to use dichotomous variables for simplicity of interpretation and to retain as many of the CTS-PC items in analyses as possible for subgroup analyses.

Control variables. We controlled for demographic variables that prior FFCWS studies indicate are related to the CTS-PC proxy variables. Control variables included maternal age based on her self-report at the time of the child's birth. Household income was assessed from mothers' reports of the total dollar amount of household income before taxes. If the mother did not provide an exact income value, she was asked to select from nine possible categories representing ranges of income. Less than $10 \%$ of the mothers were missing income data based on these two questions. For the subset of mothers who did not report income, spousal or partners' reports of household income were used. For the analyses reported here, we natural log transformed the household income variable to reduce skewedness. Marital status was treated dichotomously and dummy coded $(0=$ not married, $1=$ married) given prior work linking single parenthood and maltreatment risk (e.g., Sedlack \& Broadhurst, 1996) as well as our own multivariate analyses using FFCWS data indicating significant differences between married and unmarried parents on physical child abuse risk (Guterman, Lee, Lee, Waldfogel, \& Rathouz, 2009). Given likely covariation of ethnicity with neighborhood factors as well as with parenting styles, we first controlled for ethnicity in our overall model (African American, Hispanic, White, and other race/ethnic status), and then examined our model for differences across ethnic groups.

\section{Data analytic approach and procedures}

We tested a set of pathways linking mothers' perceptions of their neighborhood processes (social disorder, informal social control, and social cohesion), and personal variables (parenting stress and personal control) to risk for physical child abuse and neglect using structural equation modeling (SEM). All structural equation models were tested with a confirmatory 
Table 2

Structural model standardized coefficients for control variables and $R^{2}$ for latent variables.

\begin{tabular}{|c|c|c|c|c|c|c|}
\hline & $R^{2}$ & Age & Income & Married & Race: African American & Race: Hispanic \\
\hline \multicolumn{7}{|l|}{ Structural model } \\
\hline Neighborhood processes & .18 & $-.10^{* *}$ & $-.23^{* * *}$ & $-.08^{*}$ & $.24^{* * *}$ & $-.03^{*}$ \\
\hline Parenting stress & .43 & -.03 & -.02 & -.01 & .01 & -.02 \\
\hline Personal control & .14 & $-.11^{* * *}$ & $.17^{* * *}$ & .05 & $.14^{* * *}$ & -.02 \\
\hline Psychological aggression & .21 & $-.14^{* * *}$ & .03 & .03 & .05 & $-.11^{* *}$ \\
\hline Physical aggression & .20 & $-.11^{* *}$ & .05 & .05 & $.21^{* * *}$ & $-.12^{* *}$ \\
\hline Neglect & .30 & -.01 & -.05 & -.05 & -.04 & -.01 \\
\hline \multicolumn{7}{|l|}{ Race/ethnicity analysis } \\
\hline \multicolumn{7}{|l|}{ Neighborhood processes } \\
\hline White & .20 & -.08 & $-.34^{* * *}$ & $-.10^{*}$ & - & - \\
\hline African American & .08 & $-.12^{* * *}$ & $-.23^{* * *}$ & -.05 & & \\
\hline Hispanic & .10 & $-.13^{* *}$ & $-.24^{* * *}$ & -.08 & - & - \\
\hline \multicolumn{7}{|l|}{ Parenting stress } \\
\hline White & .54 & .00 & .02 & $-.11^{*}$ & & \\
\hline African American & .42 & -.04 & -.02 & .02 & & \\
\hline Hispanic & .44 & .02 & .03 & -.06 & & \\
\hline \multicolumn{7}{|l|}{ Personal control } \\
\hline White & .19 & $-.15^{* * *}$ & $.17^{* * *}$ & $.11^{* *}$ & - & - \\
\hline African American & .15 & $-.10^{* *}$ & $.16^{* * *}$ & .02 & & \\
\hline Hispanic & .12 & -.07 & $.13^{* * *}$ & $.10^{*}$ & & \\
\hline \multicolumn{7}{|l|}{ Psychological aggression } \\
\hline White & .32 & -.02 & .03 & .05 & - & - \\
\hline African American & .17 & $-.10^{*}$ & .00 & .02 & & \\
\hline Hispanic & .22 & $-.14^{* *}$ & .07 & -.07 & & \\
\hline \multicolumn{7}{|l|}{ Physical aggression } \\
\hline White & .16 & -.07 & -.02 & .10 & - & - \\
\hline African American & .12 & $-.10^{*}$ & .03 & .05 & & \\
\hline Hispanic & .16 & $-.20^{* *}$ & $.13^{*}$ & -.06 & & \\
\hline \multicolumn{7}{|l|}{ Neglect } \\
\hline White & .35 & .16 & -.05 & .03 & - & - \\
\hline African American & .30 & -.04 & -.05 & -.02 & & \\
\hline Hispanic & .29 & -.02 & -.06 & .05 & & \\
\hline
\end{tabular}

Note: Results presented for constrained model with all pathways constrained to be equal across the race/ethnicity groups standardized coefficients are presented; (-) indicates not applicable to multiple group analysis.

$p<.05$.

${ }^{* *} p<.01$.

*** $p<.001$.

latent-variable structural analysis, using weighted least squares analysis and theta parameterization in Mplus Version 5.0 (Muthén \& Muthén, 2006). We report several goodness-of-fit indices: Tucker-Lewis Index (TLI), comparative fit index (CFI), and the root-mean square error of approximation (RMSEA). The TLI and CFI are incremental fit measures that indicate the extent to which the structural model improves upon the structural null or baseline model. CFI and TLI fit indices that exceed .90 indicate that the model provides an acceptable fit to the data (Raykov, Tomer, \& Nesselroade, 1991). The RMSEA is an absolute fit index that represents the extent to which the model accounts for the observed variances and covariances. A RMSEA index at or below .06 is indicative of good fit (Hu \& Bentler, 1999). All pathways in the structural models are reported as standardized coefficients. Although we report the model $\chi^{2}$, it is important to note that $\chi^{2}$ should not be interpreted as an indicator of fit of the models or to compare between non-nested models primarily because $\chi^{2}$ is highly sensitive to sample size and is not a useful indicator of model fit when using the WLS estimator.

Latent constructs were indicated by parcels of items from the scales described in the measurement section. Parceling items to form indicators is a common procedure in structural modeling analysis, creating indicators with joint distributions that are nearer to multivariate normality (Bandalos, 2002; Kline, 2005). Latent constructs were indicated in the following manner: perceived neighborhood processes was indicated by three parcels formed by the means for each of the three scales measuring social disorder, informal social control, and social cohesion, with all scales coded so that higher scores indicated greater negative perceptions of neighborhood processes. Parenting stress was indicated by two parcels formed by the means of three items each from the parenting stress scale, with higher scores indicating greater parenting stress. Personal control was indicated by the mean score of two parcels, one with two items and one with three items, with higher scores indicating greater personal control. The outcome variables of psychological aggression, physical aggression, and neglect were indicated by individual binary items from the CTS-PC scales. Control variables were included in the model via direct effect pathways to all latent constructs in each of the models. For ease of interpretation, we report the standardized path coefficients for the control variables and the $R^{2}$ for latent variables in Table 2 rather than present them in the figures. 
Because of the large sample size and given that these relationships have not yet been explored in prior studies, we tested our models in an iterative fashion by dividing the sample into two random halves to create developmental and confirmatory samples. Using the developmental sample, we conducted all initial analyses including testing the measurement model and developing the structural model. We then tested the final model using the confirmatory sample. This strategy permits a cross-validation of the results using a sample of respondents that was essentially independent of the first sample (Kline, 2005; Rakowski et al., 1997).

To examine if the final model is equivalent for White, African American, and Hispanic respondents, we employed a multiple group SEM using the full sample of respondents. In multiple group SEM, each group (White, African American, and Hispanic) represents an independent sample and the overall model fit across the groups is assessed, thereby testing whether the models are equivalent as a function of race/ethnicity. To test model equivalence, in step 1 all variances, covariances, and factor loadings are constrained to be equal; in step 2, the factor loadings are free to vary. The $\chi^{2}$ is used to compare if the nested constrained and unconstrained models are equivalent. If the change in $\chi^{2}$ is non-significant, then the model is considered equivalent across the groups.

\section{Results}

Table 2 presents the standardized coefficients for the control variables and $R^{2}$ for latent variables as obtained from the structural model using the confirmatory sample of respondents. Table 3 presents the fit statistics and $\Delta \chi^{2}$ comparisons for the models.

Measurement model. As per the conventions of SEM, before testing the hypothesized structural model we examined the underlying measurement model in which all latent constructs were allowed to covary. The measurement model demonstrated satisfactory fit to the data: $\chi^{2}($ d.f. $=122, N=1,572)=341.24 ;$ CFI $=.93 ;$ TLI $=.94 ;$ RMSEA $=.03$.

Hypothesized structural model. Using the developmental sample, we reformulated the measurement model in terms of a structural model permitting tests of hypothesized relationships among neighborhood, parent stress, personal control and child maltreatment outcome variables. Results indicated an overall good fit of the hypothesized structural model to the developmental sample data (Table 3). We then cross-validated the results obtained with the developmental sample using the confirmatory sample. Results were nearly identical to those obtained with the developmental sample and indicated a good fit of the hypothesized structural model to the data: $\chi^{2}$ (d.f. $\left.=122, n=1,573\right)=275.64, \mathrm{CFI}=.95$, TLI $=.94$, and RMSEA $=.03$.

Direct effects. (See Fig. 1.) Mothers' perceptions of more negative neighborhood processes were predictive of lower personal control $(\beta=-.28)$, and to a lesser extent were also predictive of greater parenting stress $(\beta=.14)$. Mothers' personal control was a strong negative predictor of parenting stress $(\beta=-.60)$. Mothers' perceptions of more negative neighborhood processes were significantly directly related to psychological aggression $(\beta=.16)$ and very weakly predictive of physical $\operatorname{aggression}(\beta=.10)$. Negative neighborhood processes were not significantly directly predictive of neglect, however $(\beta=.04)$. Greater parenting stress showed a clear, direct positive relationship to greater psychological aggression $(\beta=.36)$, physical aggression $(\beta=.30)$, and neglect $(\beta=.38)$. However, greater personal control was not significantly predictive of psychological aggression $(\beta=.07)$ or physical aggression $(\beta=.10)$, but was a significant negative predictor of neglect $(\beta=-.17)$.

Indirect effects. Given the significant clear direct pathway from perceived neighborhood processes to maternal personal control, combined with the clear direct pathway from parenting stress to all three physical child maltreatment proxies, we next tested the indirect influence of personal control on physical child abuse and neglect risk via the role of parenting stress. A significant indirect effect of personal control via parenting stress was found on psychological aggression (indirect effect $\beta=-.22, p<.001$ ), physical aggression (indirect effect $\beta=-.18, p<.001$ ) and neglect (indirect effect $\beta=-.23, p<.01$ ).

Race/ethnic differences. In a final set of analyses we examined whether the model differed as a function of participants' race/ethnicity by testing the fit of the covariance matrices for African American, White, and Hispanic respondents (Fig. 2). Following the standard accepted SEM procedure for testing model equivalence, we first constrained all paths, factor loadings, and covariances to be equal (Bentler, 1989; Byrne, 1994) $\left[\chi^{2}(\mathrm{~d} . \mathrm{f} .=268, n=3,145)=756.00, \mathrm{CFI}=.91\right.$, $\mathrm{TLI}=.91$, and RMSEA $=.04$ ]. Second, we examined the fit indices for a model in which the paths among latent constructs were allowed to vary dependent on group membership (subsequently referred to as the non-constrained model) $\left[\chi^{2}\right.$ $($ d.f. $=263, n=3,145)=751.41, C F I=.91$, TLI $=.91$, and RMSEA $=.04]$. No significant change in $\chi^{2}$ between the nested con-

Table 3

Fit statistics and $\chi^{2}$ comparisons for all models.

\begin{tabular}{|c|c|c|c|c|c|c|c|}
\hline & $\chi^{2}$ & d.f. & $n$ & CFI & TLI & RMSEA & $\Delta \chi^{2}$ \\
\hline Measurement model & 341.24 & 122 & 1,572 & .93 & .94 & .03 & - \\
\hline Structural model: using developmental sample & 341.25 & 122 & 1,572 & .93 & .94 & .03 & - \\
\hline Structural model: using confirmatory sample & 275.64 & 112 & 1,573 & .95 & .94 & .03 & - \\
\hline \multicolumn{8}{|l|}{ Race/ethnicity multiple group analysis } \\
\hline Constrained Model & 756.00 & 268 & 3,145 & .91 & .91 & .04 & \\
\hline Non-constrained Model & 751.41 & 263 & 3,145 & .91 & .91 & .04 & 4.59, n.s. \\
\hline Release 2 constraints & 750.45 & 266 & 3,145 & .91 & .91 & .04 & 5.55, n.s. \\
\hline
\end{tabular}

Note: (-) $\Delta \chi^{2}$ not applicable to this analysis; $\chi^{2}$ should not be interpreted as an indicator of fit of the models or to compare between non-nested models. 


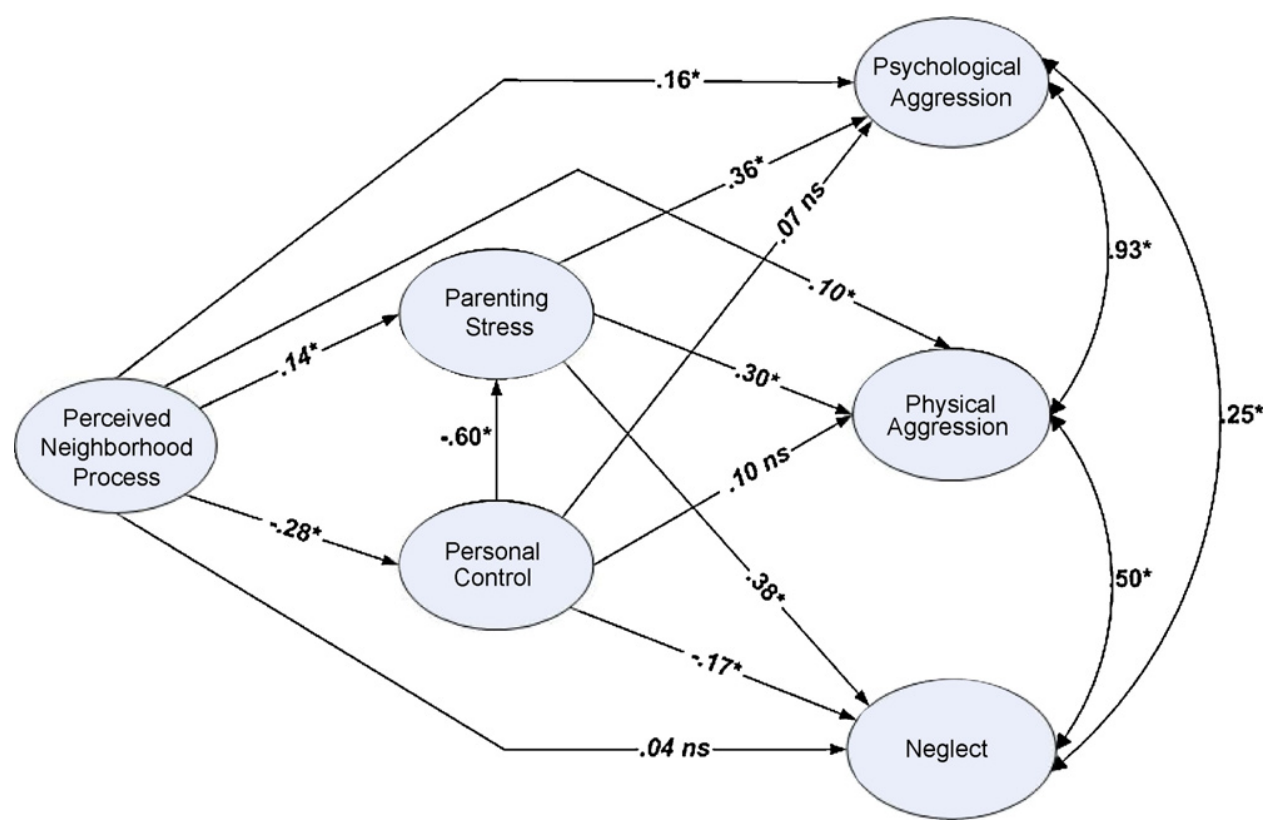

Fig. 1. Structural model using confirmatory sample $(n=1,573)$. Notes: Results shown were obtained using the confirmatory sample as described. Standardized path coefficients are reported. All paths are significant at $p<.05$ unless otherwise indicated. Model controls for maternal age at the time of the child's birth, household income, marital status, African American, White, and Hispanic race/ethnicity. Circles represent latent factors, indicated by variable parcels as described.

strained and non-constrained models was observed, indicating that the constrained model formed a better fit to the data.

In a third step, we examined the modification indices for the fully constrained model to determine whether releasing constraints on individual pathways would significantly improve the fit of the model. The modification indices suggested that releasing the pathway constraints across the race/ethnicity groups would improve the fit of the model. However,

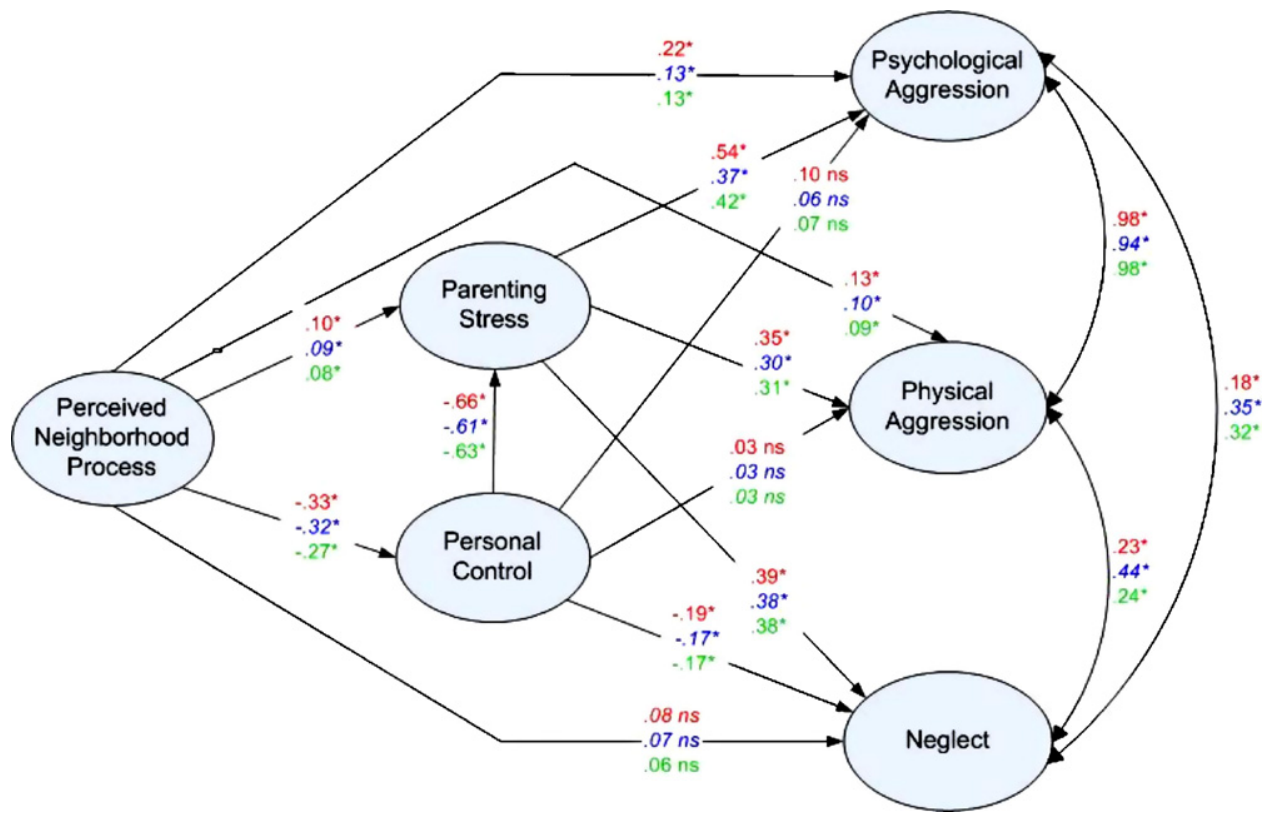

Fig. 2. Structural model moderated by race/ethnicity, fully constrained ( $n=3,145)$. Notes: Standardized path coefficients are shown. All paths are significant at $p<.05$ unless otherwise indicated. Model controls for maternal age at the time of the child's birth, household income, and marital status. Circles represent latent factors, indicated by variable parcels as described. Red font (the top series of numbers presented) is used for White families, blue italicized font (in the middle) is used for African American families, bold green font (bottom numbers) is used for Hispanic families. (For interpretation of the references to color in this figure legend, the reader is referred to the web version of the article.) 
doing so failed to result in a significantly better fitting model compared to the fully constrained model $\left[\chi^{2}\right.$ (d.f. $=266$, $n=3,145)=750.45, C F I=.91, T L I=.91$, and RMSEA $=.04]$. In summary, the results of the multiple group analysis suggest that the model is equivalent across groups and the fit of the model to the data does not vary significantly among African American, White, and Hispanic mothers. As can be seen in Fig. 2, the individual path coefficients among predictor variables across race/ethnic groups are near identical on all pathways with the exception of the negative neighborhood process-psychological aggression direct pathway ( $\beta$ for White respondents $=.22, \beta$ for African American and Hispanic respondents $=.13$ ) and the parenting stress-psychological aggression pathway ( $\beta$ for White respondents $=.54, \beta$ for African American respondents $=.37$, $\beta$ for Hispanic respondents $=.42$ ), suggesting that the overall model shows a somewhat more robust but not statistically significant predictive capacity for White respondents on psychological aggression only.

\section{Discussion}

This study employed cross-sectional data from a national birth cohort of urban families to shed light on perceived neighborhood processes and maternal risk for physical child abuse and neglect. The current findings add to the limited understanding of the pathways by which neighborhoods may shape risk for physical child maltreatment by examining relationships between mothers' self-reported perceptions of their neighborhoods, parenting stress and personal control, and risk for physical child abuse and neglect. Our findings provide support for an important role of mothers' perceptions of neighborhood processes and their risk for physical child abuse and neglect. Although we found only a mild direct link between mothers' perceptions of neighborhood processes and their risk for physical abuse (as indicated by mothers' use of psychological aggression, $\beta=.16$ and physical aggression, $\beta=.10$ ), and no direct link with mothers' self-reported neglectful behaviors, we nonetheless found a clear indirect role for mothers' perceptions of neighborhood processes on child maltreatment risk across all three proxies assessed, and across two independently drawn samples of the FFCWS study: higher perceptions of negative neighborhood processes indirectly predicted risk for both physical child abuse and neglect through mothers' lower sense of personal control, which in turn predicted greater parenting stress. Parenting stress then directly predicted mothers' greater maltreatment risk across all three proxy variables. Our findings indicate that this pattern of relationships holds up consistently across White, African-American, and Hispanic mothers examined in this study.

We further report that although mothers' personal control clearly predicts reported stress, personal control directly predicts only mothers' risk for child neglect. Personal control predicted mothers' physical abuse risk only indirectly, via parenting stresses. It should be noted that in our preliminary analyses (not shown), we examined potential bi-directionality as well as reverse directionality in the relationship between parenting stress and personal control, given earlier theoretical work that suggests a possible recursive relationship between these factors (Folkman, 1984). These preliminary analyses discerned near identical path coefficients in this relationship, regardless of the hypothesized directionality of the relationship. Given that parenting stress was observed to directly predict all three proxies of physical child maltreatment, and that neighborhood processes only directly predicted personal control and not parenting stress, we report the most interpretable model for understanding the pathways linking perceptions of neighborhood processes on maltreatment risk, indicating personal control as a predictor of stress. Unanswerable in the cross-sectional design of the present study is the degree to which some recursive patterns may be present between stress and personal control, and it therefore may be that parenting stress exerts an influence on mothers' feelings of personal control as well.

Our findings are in line with prior research reporting little observable overall direct relationship between neighborhood process factors (measured in earlier studies at the neighborhood level) and child maltreatment risk (Coulton et al., 1999; Molnar et al., 2003), as well as prior studies reporting an important role for parental stress on physical child abuse and neglect (Milner, 1998; Rodriguez \& Richardson, 2007). However, our findings augment these earlier studies by discerning an observable indirect pathway from mothers' perceptions of their neighborhood processes to physical child abuse and neglect risk via mothers' reported personal control and parenting stress. Given that several studies have reported strong correlations between objectively assessed conditions at the neighborhood level and residents perceptions of these conditions (Andresen, Malmstrom, Miller, \& Wolinsky, 2006; Perkins \& Taylor, 1996), it might be hypothesized that such objective neighborhood conditions, while not yet demonstrating a clear direct effect on maltreatment risk, may nonetheless indirectly predict risk for physical child abuse and neglect via their role in challenging parents' sense of control and stress. The FFCWS study design, with widely geographically dispersed study participants within cities and census tracts, precluded our ability to create meaningful neighborhood-level aggregated indicators of neighborhood process variables (either objective or subjective) akin to the work of Coulton et al. (1999) and Molnar et al. (2003), and therefore to directly test this hypothesis. At the same time, some have posited that attempts at assessing objective neighborhood effects on outcomes like child maltreatment using aggregated neighborhood-level indicators may fail to capture the importance of the socially constructed meaning of neighborhood conditions for parents, thus emphasizing the need to more fully incorporate an consideration of key perceptive processes and mediators (Silk et al., 2004). Indeed, this proposition is supported by evidence suggesting that individual-level perceptions of neighborhoods are more robust predictors of parenting behaviors than objective neighborhood conditions (Furstenberg et al., 1999; O'Neil et al., 2001). Thus, further work is necessary to clarify the correspondence between objective neighborhood conditions and parents' perceptions, as well as the empirical links between these objective measures and mediators of the parenting process, including high risk parenting behaviors for child maltreatment. 
In large part our findings are congruent with the proposition of Ross, Mirowsky, and Pribesh (2001) highlighting the central importance of parents' felt powerlessness as a primary mediator of stressors originating in the neighborhood, and its central role in simultaneously eroding parenting quality and amplifying or exacerbating felt stresses originating in the neighborhood environment. Drawing from learned helplessness theory (Seligman, 1975), parents challenged in their sense of personal control due to perceived inescapable aversive neighborhood conditions may retreat into a sense of helplessness in a downward spiral, and a declining sense of control in the parenting role has been shown to predict both coercive and disengaged parenting (Bugental \& Happaney, 2004; Bugental, Lewis, et al., 1999; Bugental, Lyon, Lin, McGrath, \& Bimbela, 1999).

It is important to point out that some unmeasured individual-level dispositional factors, such as those linked with mothers' mental health, may underlie parents' reported perceptions of their neighborhood conditions which may also covary with other measures in our model. Earlier work on this sample found no observable role for maternal depression on physical child abuse after controlling for parenting stress (Taylor, Guterman, Lee, \& Rathouz, 2009). However, we have not assessed a comprehensive array of such factors, and thus this also raises the methodological issue of unmeasured covariates in our model and the perennial concern over potential endogeneity biases as well, found in much of the empirical literature on neighborhood effects (Sampson et al., 2002); namely, that unmeasured dispositional factors influencing parents' selection into a particular neighborhood may simultaneously influence and therefore covary with observed child maltreatment proxies and mothers' stress and reported personal control. While we did control for maternal age at the time of the child's birth, household income, marital status, and ethnicity, such neighborhood self-selection endogeneity biases may yet be present in our analyses.

A second important limitation of the present study is its cross-sectional survey nature. Given that study predictors and outcomes were assessed at one time point, we are constrained in our ability to accurately sequence temporal directionality or to detect nuance in the interplay among study constructs. It is possible, for example, that greater felt stress and lower felt personal control may shape mothers perceptions of their neighborhoods, and as noted earlier, that stress may predict personal control. The present study design cannot definitively document the ordering of each pathway. For this initial study however, we posited the most plausible directionality among variables from an ecological standpoint. Despite these limitations, this sizeable multi-ethnic national population-based sample drawn from 20 cities across the US substantially addresses sampling bias problems endemic to clinical or problem-based (e.g., CPS referred) samples, and biases that may be attributable to US regional differences.

From a practice standpoint, consistent with work by Bugental et al. (2002) and Bugental, Lewis, et al. (1999), our findings most clearly suggest that mothers' sense of control ought to be more carefully considered as a potential key target in the prevention of physical child abuse and neglect. Augmenting the work of Bugental and colleagues, however, our findings look beyond the immediate parent-child relationship to parents' perceptions of their neighborhoods in understanding the ecological factors that shape their sense of personal control. The present study suggests a closer consideration of intervention strategies that seek to address parents' felt sense of control, not only with respect to their children, but also in relation to environmental challenges present in the neighborhood. Preventive interventions such as early home visitation may be augmented to target the enhancement parents' sense of control, especially given key findings that suggest personal control also moderates the impact of such services (Olds, Henderson, Chamberlin, \& Tatelbaum, 1986), and indeed, that the impact of such services can be demonstrably magnified when parents' perceptions of their power are enhanced (Bugental et al., 2002). Taken together, such findings suggest that enhancing parents' sense of control particularly in relation to challenges they face in their immediate social environment hold particular potential to reduce parents' felt stresses and improve parenting quality, reducing risk for physical child abuse and neglect. It appears that the ways parents experience and respond to their neighborhood conditions are indeed important to parenting. However, further evidence is necessary to elucidate the varied ways that neighborhoods, parents' experiences of their neighborhoods, and their felt stresses and control, can be effectively addressed via preventive strategies to promote more healthful rearing of children.

\section{Acknowledgements}

The authors would like to thank Erin Comartin and Eric Niklas for their research assistance on this study.

\section{References}

Abidin, R. R. (1995). Parenting stress index (3rd ed.). Odessa, FL: Psychological Assessment Resources.

Andresen, E. M., Malmstrom, T. K., Miller, D. K., \& Wolinsky, F. D. (2006). Reliability and validity of observer ratings of neighborhoods. Journal of Aging and Health, 18(1), 28-36.

Bandalos, D. L. (2002). The effects of item parceling on goodness-of-fit and parameter estimate bias in structural equation modeling. Structural Equation Modeling: A Multidisciplinary Journal, 9(1), 78-102.

Belsky, J. (1984). The determinants of parenting: A process model. Child Development, 55(1), 83-96.

Bentler, P. M. (1989). EQS structural equations program manual. Los Angeles: BMDP Statistical Software.

Browning, C. (2002). The span of collective efficacy: Extending social disorganization theory to partner violence. Journal of Marriage and Family, 64, 833-850.

Bugental, D. B., Ellerson, P. C., Lin, E. K., Rainey, B., Kokotovic, A., \& O'Hara, N. (2002). A cognitive approach to child abuse prevention. Journal of Family Psychology, 16(3), 243-258.

Bugental, D. B., \& Happaney, K. (2004). Predicting infant maltreatment in low-income families: The interactive effects of maternal attributions and child status at birth. Developmental Psychology, 40(2), 234-243. 
Bugental, D. B., Lewis, J. C., Lin, E., Lyon, J., \& Kopeikin, H. (1999). In charge but not in control: The management of teaching relationships by adults with low perceived power. Developmental Psychology, 35(6), 1367-1378.

Bugental, D. B., Lyon, J. E., Lin, E. K., McGrath, E., \& Bimbela, A. (1999). Children "tune out" in response to the ambiguous communication style of powerless adults. Child Development, $70(1), 214-230$

Byrne, B. M. (1994). Structural equation modeling with EQS and EQS-Windows: Basic concepts, applications and programming. Thousand Oaks, CA: Sage Publications.

Coulton, C. J., Crampton, D. S., Irwin, M., Spilsbury, J. C., \& Korbin, J. E. (2007). How neighborhoods influence child maltreatment: A review of the literature and alternative pathways. Child Abuse \& Neglect, 31, 1117-1142.

Coulton, C. J., Korbin, J. E., \& Su, M. (1999). Neighborhoods and child maltreatment: A multi-level study. Child Abuse E' Neglect, 23(11), 1019-1040.

Coulton, C. J., Korbin, J. E., Su, M., \& Chow, J. (1995). Community level factors and child maltreatment rates. Child Development, 66(5), $1262-1276$.

Folkman, S. (1984). Personal control and stress and coping processes: A theoretical analysis. Journal of Personality and Social Psychology, 46(4), 839-852.

Furstenberg, F. F., Cook, T. D., Eccles, J., Elder, G. H., \& Sameroff, A. (1999). Managing to make it: Urban families and adolescent success. Chicago: University of Chicago Press.

Garbarino, J. (1977). The human ecology of child maltreatment: A conceptual model for research. Journal of Marriage and the Family, 39(4), 721-735.

Garbarino, J., \& Kostelny, K. (1992). Child maltreatment as a community problem. Child Abuse E' Neglect, 16(4), 455-464.

Garbarino, J., \& Sherman, D. (1980). High risk neighborhoods and high risk families: The human ecology of child maltreatment. Child Development, 51(1), $188-198$.

Geis, K. J., \& Ross, C. E. (1998). A new look at urban alienation: The effect of neighborhood disorder on perceived powerlessness. Social Psychology Quarterly, $61,236-242$

Guterman, N. B., Lee, Y., Lee, S. J., Waldfogel, J., \& Rathouz, P. J. (2009). Fathers and maternal risk for physical child abuse. Child Maltreatment, 14(3), 277-290.

Gutman, L. M., McLoyd, V. C., \& Tokoyawa, T. (2008). Financial strain, neighborhood, stress, parenting behaviors, and adolescent adjustment in urban African American families. Journal of Research on Adolescence, 154(4), 425-449.

Haskett, M. E., Ahern, L. S., Ward, C. S., \& Allaire, J. C. (2006). Factor structure and validity of the Parenting Stress Index-Short Form. Journal of Clinical Child E Adolescent Psychology, 35(2), 302-312.

Hill, T. D., Ross, C. E., \& Angel, R. J. (2005). Neighborhood disorder, psychophysiological distress, and health. Journal of Health and Social Behavior, 46, $170-186$.

Hu, L., \& Bentler, P. M. (1999). Cutoff criteria for fit indexes in covariance structure analysis: Conventional criteria versus new alternatives. Structural Equation Modeling, 6(1), 1-55.

King, G., Reece, R., Bendel, R., \& Patel, V. (1998). The effects of sociodemographic variables, training, and attitudes on the lifetime reporting practices of mandates reporters. Child Maltreatment, 3, 276-283.

Kline, R. B. (2005). Principles and practice of structural equation modeling (2nd ed.). New York: Guilford.

Kohen, D. E., Leventhal, T., Dahinten, V. S., \& McIntosh, C. N. (2005). Neighborhood disadvantage: Pathways of effects for young children. Child Development, $79(1), 156-169$

Milner, J. S. (1998). Individual and family characteristics associated with intrafamilial child physical and sexual abuse. In P. K. Trickett, \& C. J. Schellenbach (Eds.), Violence against children in the family and the community (pp. 141-170). Washington, DC: American Psychological Association.

Molnar, B. E., Buka, S. L., Brennan, R. T., Holton, J. K., \& Earls, F. (2003). A multilevel study of neighborhoods and parent-to-child physical aggression: Results from the Project on Human Development in Chicago neighborhoods. Child Maltreatment, 8(2), 84-97.

Muthén, B., \& Muthén, L. K. (2006). Mplus (Version 5.0) [Computer software]. Los Angeles: Muthén \& Muthén.

National Research Council. (1993). Understanding child abuse and neglect. Washington, DC: National Academy Press.

O'Neil, R., Parke, R. D., \& McDowell, D. J. (2001). Objective and subjective features of children's neighborhoods: Relations to parental regulatory strategies and children's social competence. Applied Developmental Psychology, 22, 135-155.

Olds, D., Henderson, C. R., Chamberlin, R., \& Tatelbaum, R. (1986). Preventing child abuse and neglect: A randomized trial of nurse home visitation. Pediatrics, $78(1), 65-78$

Pearlin, L. I., \& Schooler, C. (1978). The structure of coping. Journal of Health and Social Behavior, 19(1), 2-21.

Perkins, D. D., \& Taylor, R. B. (1996). Ecological assessments of community disorder: Their relationship to fear of crime and theoretical implications. American Journal of Community Psychology, 24(1), 63-104.

Rakowski, W., Andersen, M. R., Stoddard, A. M., Urban, N., Rimer, B., Lane, D. S., Fox, S. A., \& Costanza, M. E. (1997). Confirmatory analysis of opinions regarding the pros and cons of mammography. Health Psychology, $16,5$.

Raykov, T., Tomer, A., \& Nesselroade, J. R. (1991). Reporting structural equation modeling results in Psychology and Aging: Some proposed guidelines. Psychology and Aging, 6(4), 499-503.

Reichman, N., Teitler, J., Garfinkel, I., \& McLanahan, S. (2001). Fragile families: Sample and design. Children and Youth Services Review, 32(4/5), 303-326.

Rodriguez, C. M., \& Green, A. J. (1997). Parenting stress and anger expression as predictors of child abuse potential. Child Abuse E' Neglect, $21,367-377$.

Rodriguez, C. M., \& Richardson, M. J. (2007). Stress and anger as contextual factors and preexisting cognitive schemas: Predicting parental child maltreatment risk. Child Maltreatment, 12(4), 325-337.

Ross, C. E., Mirowsky, J., \& Pribesh, S. (2001). Powerlessness and the amplification of threat: Neighborhood disadvantage, disorder, and mistrust. American Sociological Review, 66, 568-591.

Sampson, R. J., Morenoff, J. D., \& Gannon-Rowley, T. (2002). Assessing "neighborhood effects": Social processes and new directions in research. Annual Review of Sociology, 28, 443-478.

Sampson, R. J., Raudenbush, S. W., Stephen, W., \& Earls, F. (1997). Neighborhoods and violent crime: A multilevel study of collective efficacy. Science, 277(5328), 918-924.

Sedlack, A., \& Broadhurst, D. D. (1996). The third national incidence study of child abuse and neglect: Final report. Washington, DC: U.S. Government Printing Office.

Seligman, M. E. P. (1975). Helplessness: On depression, development and death. San Francisco, CA: W.H. Freedman.

Silk, J. S., Sessa, F. M., Morris, A. S., Steinberg, L., \& Avenevoli, S. (2004). Neighborhood cohesion as a buffer against hostile maternal parenting. Journal of Family Psychology, 18(1), 135-146.

Socolar, R. S., Runyan, D. K., \& Amaya-Jackson, L. (1995). Methodological and ethical issues related to studying child maltreatment. Journal of Family Issues, $16,565-586$

Straus, M. A., Hamby, S. L., Finkelhor, D., Moore, D. W., \& Runyan, D. (1998). Identification of child maltreatment with the Parent-Child Conflict Tactics Scales: Development and psychometric data for a national sample of American parents. Child Abuse E Neglect, 22(4), 249-270.

Taylor, C. T., Guterman, N. B., Lee, S. J., \& Rathouz, P. J. (2009). Intimate partner violence, maternal stress, nativity, and risk for maternal maltreatment of young children. American Journal of Public Health, 99(1), 1-8. 